\title{
A SIMULATION OF THE THERMAL STATE OF HEAVILY LOADED TRIBO-UNITS AND ITS EVALUATION
}

Yu. Rozhdestvensky, South Ural State University, Chelyabinsk, Russian Federation, ruv@susu.ac.ru,

E. Zadorozhnaya, South Ural State University, Chelyabinsk, Russian Federation, elena-nea@rambler.ru

The thermal state of the elements of heavily loaded tribo-units is among the most important parameters affecting their performance. The temperature of the lubricating layer of bearings determines to a large extent their load-bearing capacity. The heat balance equation used to estimate the temperature of friction pairs fails to yield the temperature fields and the regions of their maximum values. This fact makes our problem important. We examine a mathematical model and a method for calculating the thermal state and thermohydrodynamic characteristics of heavily loaded sliding bearings, taking into account the non-Newtonian properties of the fluid as well as the heat exchange processes between the lubricating layer and the surrounding moving surfaces of tribo-units. To solve the energy equation, we propose to use finite difference approximation methods. To create the difference analogs of the energy equations for some structural elements and thin lubricant layers, we use the Pismen-Reckford scheme of implicit alternating directions. We present the calculated hydromechanical characteristics of the connecting rod bearing of a heat engine. We obtain three-dimensional distributions of temperature in the lubricant.

The results show that, if we allow for convective heat transfer in the radial direction, the processes of heat exchange between the lubricating layer and the surrounding moving surfaces enable us to determine more accurately the mean lubricant temperature and the thermal stress of a tribo-unit as a whole. Our method can be used to assess the performance and efficiency of heavily loaded tribo-units of piston and rotary machines.

Keywords: bearing; generalized energy equation; partial differential equations; boundary value problems.

\section{Introduction}

The problem of improving the reliability and durability of friction units in machines has always been a major challenge of modern engineering. The rising power of thermal machines and the rising demands for durability and fuel efficiency result in the increased loading of tribo-units (TU). In this regard, it is important to choose methods for solving concrete problems, as well as to create methods for physical and mathematical modeling of friction and the wear of friction units.

Modern mechanical engineering is based on advanced technologies. Innovative computational methods and computer technologies allow engineers and researchers to simulate and calculate TU for different machines and consider a number of design, operation, and other parameters affecting the performance of friction units. However, the thermal processes occurring in a heavily loaded bearing are of great importance for understanding the performance of friction units. Usually they are treated on the basis of a solution to the generalized energy equation (heat transfer equation) for a thin layer 
of viscous incompressible fluid between two arbitrarily moving surfaces. This equation involves both convective heat transfer in the lubricant and heat transfer by conduction.

Thus, our research aims at developing models and algorithms to solve the problems of TU dynamics taking into account the temperature distribution in the lubricating layer, the non-Newtonian properties of the lubricant, and the geometry of friction surfaces bounding the thin lubricating layer.

\section{Solution}

To solve the stated problem, consider a circular cylindrical radial bearing, where the bearing (bushing) 1 and the journal 2 rotate round the axes $O_{i} Z_{i}$, with $i=1,2$ passing through their centers $O_{i}$ with absolute angular velocities $\omega_{i}$. The lubricating layer of nonNewtonian fluid is bounded by the surfaces of the bearing and the journal (see Fig. 1). Here $x=r \varphi$ with $r \approx r_{1} \approx r_{2}$, where $r_{1}=r_{1}(\varphi, t)$ and $r_{2}=r_{2}(\varphi, t)$ are the radii of bushing (the inner surface) and the journal; $\varphi$ is the angular coordinate measured from the axis $O_{1} X_{1}$ rigidly fastened to the bushing. The axes $O x$ and $O z$ of the coordinate system $O x y z$, in which we consider the processes in the lubricating layer, lie in the plane of the reference surface, and the axis $O y$ is perpendicular to it. The external force $F(t)$ depending on time $t$ acts on the pin in the plane $O x y$ in the central cross section $z=0$.

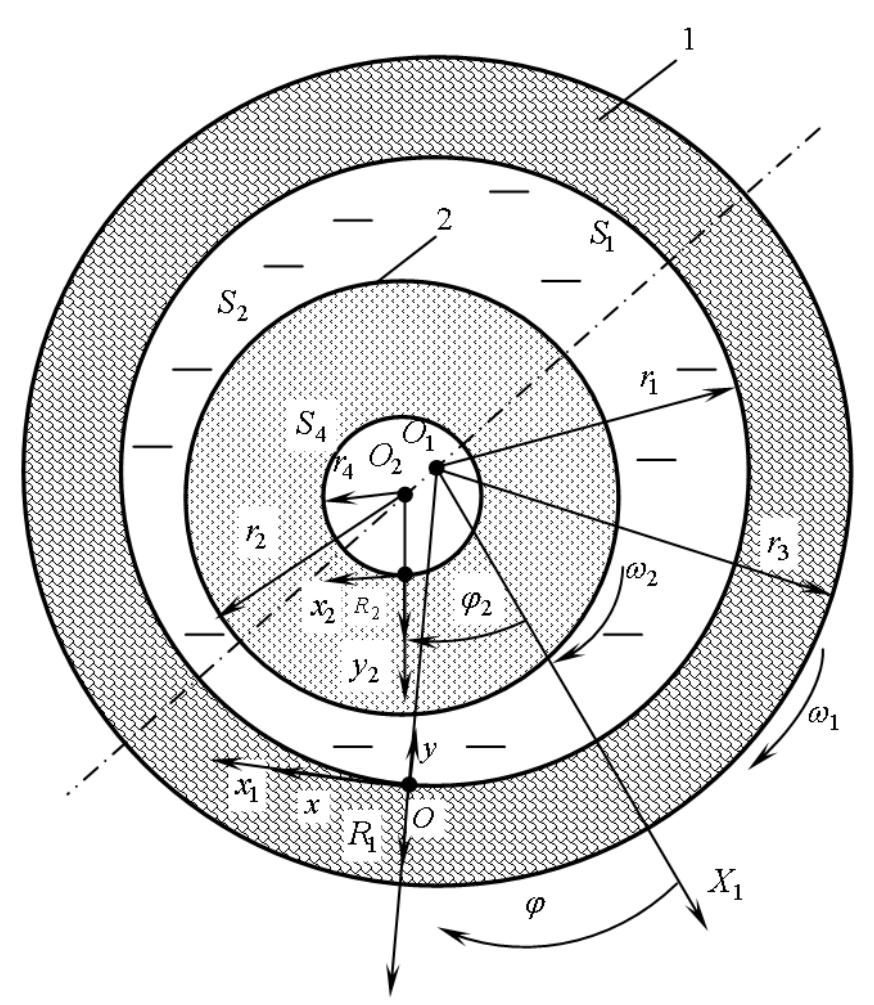

Fig. 1. Coordinate systems for solving the heat problem

To determine the field of hydrodynamic pressures in the thin lubricating layer, use the generalized Reynolds equation [1-3]. For a non-Newtonian fluid we can write it as 


$$
\begin{gathered}
\frac{\partial}{\partial \varphi}\left[\bar{h}^{n+2}\left(\bar{\phi}_{2}-\frac{\bar{\phi}_{1}^{2}}{\overline{\phi_{0}}}\right) \bar{\rho} \frac{\partial \bar{p}}{\partial \varphi}\right]+\frac{\partial}{\partial \bar{z}}\left[\bar{h}^{n+2}\left(\bar{\phi}_{2}-\frac{\bar{\phi}_{1}^{2}}{\bar{\phi}_{0}}\right) \bar{\rho} \frac{\partial \bar{p}}{\partial \bar{z}}\right]= \\
=\frac{\partial}{\partial \varphi}\left[\bar{\omega}_{21} \bar{\rho} \bar{h}\left(1-\frac{\bar{\phi}_{1}}{\bar{\phi}_{0}}\right)\right]+\frac{\partial}{\partial \bar{t}}(\bar{\rho} \bar{h})
\end{gathered}
$$

where $\varphi$ and $z$ are the angular and axial coordinates; $\bar{\rho}=\rho / \rho_{0}$ is the dimensionless density; $\rho_{0}$ is the density of the Newtonian fluid; $\bar{p}=\left(p-p_{a}\right) \psi^{2} / \mu_{0} \omega_{0}, \psi=h_{0} / r_{2}, \bar{z}=z / r_{2}$ with $-a \leq \bar{z} \leq a$, and $\bar{t}=\omega_{0} t$ are the dimensionless hydrodynamic pressure, relative radial clearance, width coordinate of the bearing, and time; $h_{0}$ is the radial adjusting clearance; $a=B / D$ is the relative width of the bearing; $\mu_{0}$ is the characteristic viscosity of the lubricant; $p_{a}$ is atmospheric pressure; $B, D=2 r_{2}$, and $r_{2}$ are respectively the width, diameter, and radius of the pin; $\bar{\omega}_{21}=\left(\omega_{2}-\omega_{1}\right) / \omega_{0}$ is the dimensionless angular velocity of the pin. The dimensionless thickness $\bar{h}$ of the film and its derivative $\partial \bar{h} / \partial \bar{t}$ are defined as

$$
\bar{h}=1-\chi \cos (\varphi-\delta), \quad \partial \bar{h} / \partial \bar{t}=-\dot{\chi} \cos (\varphi-\delta)-\chi \dot{\delta} \cos (\varphi-\delta),
$$

where $\chi$ is the relative eccentricity and $\delta$ is the angle of relative position of the center line, furthermore,

$$
\bar{\phi}_{k}=\int_{0}^{\bar{h}} \bar{y}^{k} / \bar{\mu}^{*} d \bar{y}, k=0,1,2 \text {, }
$$

where $\bar{\mu}^{*}$ is the dimensionless non-Newtonian viscosity of the lubricant, which is a function of shear rate, temperature, and pressure; finally, $\bar{y}$ is the dimensionless coordinate across the lubricating layer.

Use a multigrid method to integrate (1) with the Swift-Stieber boundary conditions, taking into account the presence of sources of lubrication (holes or grooves) on the friction surfaces [4]:

$$
\begin{gathered}
\bar{p}(\varphi, \bar{z}= \pm a)=0, \bar{p}(\varphi, \bar{z})=\bar{p}(\varphi+2 \pi, \bar{z}), \bar{p}(\varphi, \bar{z}) \geq 0 \text { on }(\varphi, \bar{z}) \in \Omega_{s} \\
\bar{p}(\varphi, \bar{z})=\bar{p}_{s}, S=1,2 \ldots, S^{*}
\end{gathered}
$$

where the region $\Omega_{s}$ stands for a source of lubricant, in which the pressure is constant and equals the supply pressure $\bar{p}_{s}$, while $S^{*}$ is the number of sources of lubrication. Express the dependence of viscosity on the shear rate and pressure as $[5,6]$ :

$$
\bar{\mu}^{*}=\left\{\begin{array}{l}
\bar{\mu}_{1} \cdot \exp (\beta \cdot \bar{p}), \quad I_{2}<10^{4} c^{-1} \\
\bar{\mu} \cdot I_{2}^{(n-1) / 2} \cdot \exp (\beta \cdot \bar{p}), \quad 10^{4}<I_{2}<10^{6} c^{-1} \\
\bar{\mu}_{2} \cdot \exp (\beta \cdot \bar{p}), \quad I_{2}>10^{6} c^{-1}
\end{array}\right.
$$

The parameter $n$ characterizes the degree of non-Newtonian behavior and $\beta$ is the pressureviscosity coefficient of the lubricant, which is a function of temperature.

According to the model (2) in region 1 (with $I_{2}<10^{4} c^{-1}$ ) the lubricant behaves as a Newtonian fluid with viscosity $\mu_{1}\left(T_{p}, p\right)$. In region 2 (with $10^{4}<I_{2}<10^{6} c^{-1}$ ) viscosity decreases following a power law. In region 3 (with $I_{2}>10^{6} \mathrm{c}^{-1}$ ) the lubricant is considered as a Newtonian fluid with viscosity $\mu_{2}\left(T_{p}, p\right)$.

Let us express in a dimensionless form the equations for the velocities of a volume element of the lubricant and their derivatives:

$$
\bar{V}_{x}=\frac{\bar{\phi}_{0 y}}{\bar{\phi}_{0}} \bar{\omega}_{21}+\left(\bar{\phi}_{1 y}-\frac{\bar{\phi}_{1}}{\bar{\phi}_{0}} \bar{\phi}_{0 y}\right) \bar{h}^{n+1} \cdot \frac{\partial \bar{p}}{\partial \varphi}, \bar{V}_{z}=\left(\bar{\phi}_{1 y}-\frac{\bar{\phi}_{1}}{\bar{\phi}_{0}} \bar{\phi}_{0 y}\right) \bar{h}^{n+1} \cdot \frac{1}{a} \frac{\partial \bar{p}}{\partial \bar{z}}
$$




$$
\begin{gathered}
\bar{V}_{y}=\bar{h} \psi\left[\frac{1}{\bar{h}} \frac{\partial \bar{h}}{\partial \varphi}\left(\bar{V}_{x} \bar{y}-\bar{q}_{x y}\right)-\frac{\partial \bar{q}_{x y}}{\partial \varphi}-\frac{1}{a} \frac{\partial \bar{q}_{z y}}{\partial \varphi}\right], \bar{\phi}_{k y}=\int_{h_{1}}^{\bar{y}} \frac{\bar{y}^{k} d \bar{y}}{\bar{\mu}^{*}}, \quad k=0,1,2, \\
\frac{\partial \bar{V}_{x}}{\partial \bar{y}}=\frac{1}{\bar{\mu}^{*}} \cdot\left[\frac{\bar{\omega}_{21}}{\bar{\phi}_{0}}+\bar{h}^{n+1}\left(\bar{y}-\frac{\bar{\phi}_{1}}{\bar{\phi}_{0}}\right) \frac{\partial \bar{p}}{\partial \varphi}\right], \frac{\partial \bar{V}_{z}}{\partial \bar{y}}=\frac{1}{\bar{\mu}^{*}} \cdot \bar{h}^{n+1}\left(\bar{y}-\frac{\bar{\phi}_{1}}{\bar{\phi}_{0}}\right) \frac{\partial \bar{p}}{\partial \bar{z}}
\end{gathered}
$$

Write down the flux of the lubricant across the sections of unit length along the $x$ and $z$ coordinates as

$$
\bar{q}_{x y}=\int_{0}^{\bar{y}} \bar{V}_{x} d \bar{y} ; \bar{q}_{z y}=\int_{0}^{\bar{y}} \bar{V}_{z} d \bar{y} .
$$

The generalized energy equation (heat transfer equation) for the lubricating layer of viscous incompressible fluid, accounting for small thermal conductivity along the $x$ and $z$ axes, is $[6-8]$

$$
\rho c_{0} \frac{\partial T}{\partial t}+\rho c_{0}\left(V_{x} \frac{\partial T}{\partial x}+V_{y} \frac{\partial T}{\partial y}+V_{z} \frac{\partial T}{\partial z}\right)-\lambda_{0} \frac{\partial^{2} T}{\partial y^{2}}=D s,
$$

where $\rho, c_{0}$, and $\lambda_{0}$ are the density, specific heat, and thermal conductivity of the lubricant (taken to be constants); $V_{x}, V_{y}$, and $V_{z}$ are the components of the velocity vector of a volume element of the lubricant; $D s=\mu^{*}\left[\left(\frac{\partial V_{x}}{\partial y}\right)^{2}+\left(\frac{\partial V_{z}}{\partial y}\right)^{2}\right]$ is a dissipative function.

Introduce the following notation: $\bar{T}=T / T_{0}$ is the dimensionless temperature at a point of the lubricating layer; $T_{0}$ is characteristic temperature; $\bar{y}=y / h, \bar{h}=h / h_{0}^{*}, \psi=h_{0}^{*} / r$, $\bar{V}_{x}=V_{x} / \omega_{0} r, \bar{V}_{y}=V_{y} / \omega_{0} r, \bar{V}_{z}=V_{z} / \omega_{0} r, \bar{\mu}=\mu / \mu_{0}$, where $h_{0}^{*}$ is the characteristic thickness of the film for the central position of the pin, $P e=\rho_{0} \bar{\rho} c_{0} \omega_{0} \Delta_{0}^{2} / \lambda_{0}$ is the Peclet number, $k_{T}=\rho_{0} \bar{\rho} c_{0} T_{0} \psi^{2} /\left(\omega_{0} \mu_{0}\right)$. In this case, we can write the energy equation for the lubricating film of the bearing with non-Newtonian lubricant in dimensionless form as

$$
\frac{\partial \bar{T}}{\partial \bar{t}}=-\bar{V}_{x} \frac{\partial \bar{T}}{\partial \varphi}-\bar{D} \frac{\partial \bar{T}}{\partial \bar{y}}-\bar{V}_{z} \frac{\partial \bar{T}}{\partial \bar{z}}+\frac{1}{P e} \cdot \frac{1}{\bar{h}^{2}} \cdot \frac{\partial^{2} \bar{T}}{\partial \bar{y}^{2}}+\frac{1}{k_{T}} \cdot \frac{1}{\bar{h}^{n+1}} \bar{D} s,
$$

where

$$
\bar{D}=-\frac{1}{\bar{h}}\left[\bar{y} \frac{\partial \bar{h}}{\partial \bar{t}}+\frac{\partial\left(\bar{h} \bar{q}_{x y}\right)}{\partial \varphi}+\bar{h} \frac{\partial \bar{q}_{z y}}{\partial \bar{z}}\right] .
$$

This differential equation is linear with respect to derivatives. The coefficients of the convective terms depend on $\varphi, \bar{y}, \bar{z}$, and $\bar{t}$. The equation is parabolic in time, and so we impose initial and boundary conditions.

Determine the distribution $\bar{T}_{1}\left(\varphi_{1}, R_{2}, \bar{t}\right)$ of temperature in the bushing, where $R_{2}$ is the radial coordinate (see Fig. 1), by solving the equation for transient heat flow, which in cylindrical coordinates and dimensionless variables becomes

$$
\frac{\partial \bar{T}_{1}}{\partial \bar{t}}=\bar{\alpha}_{1}\left(\frac{\partial^{2} \bar{T}_{1}}{\partial \bar{R}_{1}^{2}}+\frac{1}{\overline{R_{1}}} \frac{\partial \bar{T}_{1}}{\partial \bar{R}_{1}}+\frac{1}{\bar{R}_{1}^{2}} \frac{\partial^{2} \bar{T}_{1}}{\partial \varphi_{1}^{2}}\right)
$$

Here $\bar{R}_{1}=R_{1} / r$ and $\bar{T}_{1}=T_{1} / T_{0}$; furthermore, $\bar{\alpha}_{1}=\lambda_{1} /\left(c_{1} \rho_{1} r^{2} \omega_{0}\right)$ is the dimensionless coefficient of heat transfer from the bushing to the environment, with $r_{1} \approx r_{2} \approx r$, while $\rho_{1}, c_{1}$, and $\lambda_{1}$ are the density, specific heat, and thermal conductivity of the material of the bushing. 
Introduce coordinate system $O x_{1} y_{1} z_{1}$ and dimensionless variables

$$
\bar{y}_{1}=\left(R_{1}-r_{1}\right) /\left(r_{3}-r_{1}\right)=\left(\bar{R}_{1}-1\right) /\left(\bar{r}_{3}-1\right), \quad \bar{r}_{3}=r_{3} / r,
$$

where $r_{1}$ and $r_{3}$ are the radii of the inner and outer surfaces of the bushing. Then (7) becomes

$$
\frac{\partial \bar{T}_{1}}{d \bar{t}}=\bar{\alpha}_{1}\left(\frac{1}{\left(\bar{r}_{3}-1\right)^{2}} \frac{\partial^{2} \bar{T}_{1}}{\partial \bar{y}_{1}^{2}}+\frac{1}{\left[1+\left(\bar{r}_{3}-1\right) \bar{y}_{1}\right]\left(\bar{r}_{3}-1\right)} \frac{\partial \bar{T}_{1}}{\partial \bar{y}_{1}}+\frac{1}{\left[1+\left(\bar{r}_{3}-1\right) \bar{y}_{1}\right]^{2}} \frac{\partial^{2} \bar{T}_{1}}{\partial \varphi^{2}}\right) .
$$

Similarly, determine the distribution $\bar{T}_{2}\left(\varphi_{2}, R_{2}, \bar{t}\right)$ of temperature in the pin by solving the equation for transient heat flow, which in cylindrical coordinates and dimensionless variables becomes

$$
\frac{\partial \bar{T}_{2}}{\partial \bar{t}}=\bar{\alpha}_{2}\left(\frac{\partial^{2} \bar{T}_{2}}{\partial \bar{R}_{2}^{2}}+\frac{1}{\overline{R_{2}}} \frac{\partial \bar{T}_{2}}{\partial \bar{R}_{2}}+\frac{1}{\bar{R}_{2}^{2}} \frac{\partial^{2} \bar{T}_{2}}{\partial \varphi_{2}^{2}}\right) .
$$

Here $\bar{R}_{2}=R_{2} / r$ and $\bar{T}_{2}=T_{2} / T_{0}$; furthermore, $\bar{\alpha}_{2}=\lambda_{2} /\left(c_{2} \rho_{2} r^{2} \omega_{0}\right)$ is the dimensionless coefficient of heat transfer from the pin to the environment, while $\rho_{2}, c_{2}$, and $\lambda_{2}$ are the density, specific heat, and thermal conductivity of the material of the pin.

In coordinate system $\mathrm{Ox}_{2} y_{2} z_{2}$ introduce the dimensionless variables

$$
\bar{y}_{2}=\left(R_{2}-r_{4}\right) /\left(r_{2}-r_{4}\right)=\left(\bar{R}_{2}-\bar{r}_{4}\right) /\left(1-\bar{r}_{4}\right), \quad \bar{r}_{4}=r_{4} / r,
$$

where $r_{4}$ is the radius of the inner surface of the bushing. Then (9) becomes

$$
\frac{\partial \bar{T}_{2}}{d \bar{t}}=\bar{\alpha}_{2}\left(\frac{1}{\left(1-\bar{r}_{4}\right)^{2}} \frac{\partial^{2} \bar{T}_{2}}{\partial \bar{y}_{2}^{2}}+\frac{1}{\left[\bar{r}_{4}+\left(1-\bar{r}_{4}\right) \bar{y}_{2}\right]\left(1-\bar{r}_{4}\right)} \frac{\partial \bar{T}_{2}}{\partial \bar{y}_{2}}+\frac{1}{\left[\bar{r}_{4}+\left(1-\bar{r}_{4}\right) \bar{y}_{2}\right]^{2}} \frac{\partial^{2} \bar{T}_{2}}{\partial \varphi^{2}}\right) .
$$

Let us state the boundary conditions to integrate the heat subproblem (5), (8), and (10). Since the temperatures of the lubricant and the bushing are periodic in the circumferential direction, we have

$$
\bar{T}(\varphi, \bar{y}, \bar{t})=\bar{T}(\varphi+2 \pi, \bar{y}, \bar{t}), \quad \bar{T}_{1}\left(\varphi, \bar{R}_{1}, \bar{t}\right)=\bar{T}_{1}\left(\varphi+2 \pi, \bar{R}_{1}, \bar{t}\right) .
$$

On the outer surface of the bushing assume the free convection hypothesis

$$
\left.\frac{\partial \bar{T}_{1}}{\partial \bar{R}}\right|_{\bar{R}=\bar{r}_{3}}=\frac{\alpha_{1} r}{\lambda_{1}}\left(\left.\bar{T}_{1}\right|_{\bar{R}=\bar{r}_{3}}-\bar{T}_{c}\right) .
$$

On the common surface of the lubricant and the bushing impose the continuity condition for the heat flux (the coupling condition)

$$
\left.\frac{\partial \bar{T}_{1}}{\partial \overline{y_{1}}}\right|_{\bar{y}_{1}=0}=-\left.\left(\bar{r}_{3}-1\right) \frac{\lambda_{0}}{\lambda_{1} \bar{h} \psi} \frac{\partial \overline{T_{1}}}{\partial \bar{y}}\right|_{\bar{y}=0} .
$$

On the surfaces of the lubricant shared with the sleeve and spike impose the equal temperature condition

$$
\bar{T}(\varphi, \bar{y}=0, \bar{t})=\overline{T_{1}}\left(\varphi, \bar{y}_{1}=0, \bar{t}-\bar{t}_{c}\right), \quad \bar{T}(\varphi, \bar{y}=1, \bar{t})=\bar{T}_{2}\left(\bar{t}-\bar{t}_{c}\right) .
$$




\section{Finite-Difference Approximation for the Equations of the Thermal Subproblem}

Express (5), (8), and (10) in the dimensionless form:

$$
\begin{aligned}
& \frac{\partial \bar{T}}{\partial \bar{t}}=K_{1} \frac{\partial \bar{T}}{\partial \varphi}+K_{2} \frac{\partial \bar{T}}{\partial \bar{y}}+K_{3} \frac{\partial^{2} \bar{T}}{\partial \bar{y}^{2}}+K_{4}, \\
& \frac{\partial \bar{T}_{1}}{\partial \bar{t}}=K_{5} \frac{\partial^{2} \bar{T}_{1}}{\partial \bar{y}_{1}^{2}}+K_{6} \frac{\partial \bar{T}_{1}}{\partial \bar{y}_{1}}+K_{7} \frac{\partial^{2} \bar{T}_{1}}{\partial \varphi^{2}} \\
& \frac{\partial \bar{T}_{2}}{\partial \bar{t}}=K_{8} \frac{\partial^{2} \bar{T}_{2}}{\partial \bar{y}_{2}^{2}}+K_{9} \frac{\partial \bar{T}_{2}}{\partial \bar{y}_{2}}+K_{10} \frac{\partial^{2} \bar{T}_{2}}{\partial \varphi^{2}} .
\end{aligned}
$$

Here we put

$$
\begin{gathered}
\bar{T}=\bar{T}^{\prime}, \\
K_{1}(\varphi, \bar{y}, \bar{t})=-\bar{V}_{x}, K_{2}(\varphi, \bar{y}, \bar{t})=-\bar{D}, K_{3}(\varphi)=\frac{1}{P e \cdot \bar{h}^{2}}, K_{4}(\varphi)=\frac{D s}{k_{T} \cdot \bar{h}^{n+1}}, \\
K_{5}=\bar{\alpha}_{1} \frac{1}{\left(\bar{r}_{3}-1\right)^{2}}, K_{6}\left(\bar{y}_{1}\right)=\frac{\bar{\alpha}_{1}}{\left(\left(\bar{r}_{3}-1\right)\right)\left[1+\left(\bar{r}_{3}-1\right) \bar{y}_{1}\right]}, K_{7}\left(\bar{y}_{1}\right)=\frac{\bar{\alpha}_{1}}{\left[1+\left(\bar{r}_{3}-1\right) \bar{y}_{1}\right]^{2}}, \\
K_{8}=\bar{\alpha}_{2} \frac{1}{\left(1-\bar{r}_{4}\right)^{2}}, K_{9}\left(\bar{y}_{2}\right)=\frac{\bar{\alpha}_{2}}{\left(1-\bar{r}_{4}\right)\left[\bar{r}_{4}+\left(1-\bar{r}_{4}\right) \bar{y}_{2}\right]}, K_{10}\left(\bar{y}_{2}\right)=\frac{\bar{\alpha}_{2}}{\left[\bar{r}_{4}+\left(1-\bar{r}_{4}\right) \bar{y}_{2}\right]^{2}} .
\end{gathered}
$$

For generality, we also replaced $\bar{y}, \bar{y}_{1}, \bar{y}_{2} \Rightarrow y$ and $\bar{T}, \bar{T}_{1}, \bar{T}_{2} \Rightarrow T$.

Express the system of equations for the two-dimensional distribution of temperature in the lubricating layer, bushing, and pin in the operator form

$$
T_{\bar{t}}+A \cdot L_{\varphi}(T)+B \cdot L_{y}(T)=C \cdot L_{\varphi \varphi}(T)+D \cdot L_{y y}(T)+E,
$$

where

$$
\begin{gathered}
T=\left[\begin{array}{c}
\bar{T} \\
\bar{T}_{1} \\
\bar{T}_{2}
\end{array}\right], A=\left[\begin{array}{c}
-K_{1} \\
0 \\
0
\end{array}\right], B=\left[\begin{array}{l}
-K_{2} \\
-K_{6} \\
-K_{9}
\end{array}\right], C=\left[\begin{array}{l}
0 \\
K_{7} \\
K_{10}
\end{array}\right], D=\left[\begin{array}{l}
K_{3} \\
K_{5} \\
K_{8}
\end{array}\right], E=\left[\begin{array}{l}
K_{4} \\
0 \\
0
\end{array}\right], \\
T_{\bar{t}}=\frac{\partial \bar{T}}{\partial \bar{t}}, L_{\varphi}(T)=\frac{\partial \bar{T}}{\partial \varphi}, L_{y}(T)=\frac{\partial \bar{T}}{\partial \bar{y}}, L_{\varphi \varphi}(T)=\frac{\partial^{2} \bar{T}}{\partial \varphi^{2}}, L_{y y}(T)=\frac{\partial^{2} \bar{T}}{\partial \bar{y}^{2}} .
\end{gathered}
$$

For the finite difference approximation, denote by $l_{\varphi}=2 \pi, l_{z}=2$, and $l_{y}=1$ the lengths of TU elements along the coordinates $\varphi, z$, and $y$ and choose the grid with the coordinates $\left(\varphi_{i}, \bar{z}_{j}, \bar{y}_{k}, \bar{t}_{n}\right)$ of the nodes, where

$$
\begin{gathered}
\varphi_{i}=(i-1) \Delta_{\varphi}\left(i=1,2, \ldots N, \Delta_{\varphi}=l_{\varphi} /(N-1)\right) \\
\bar{z}_{j}=-1+(j-1) \Delta_{z}\left(j=1,2, \ldots M, \Delta_{z}=l_{z} /(M-1)\right) ; \\
\bar{y}_{k}=(k-1) \Delta_{y}\left(k=1,2 \ldots K, \Delta_{y}=l_{y} /(K-1)\right) ; \\
\bar{t}_{n}=n \Delta_{\bar{t}}(n=0,1,2, \ldots) .
\end{gathered}
$$


The step and the number of mesh elements depend on the direction: $(N+1)=96$, $(M+1)=25$, and $K=20$. At each time step we introduce arrays with elements. The one-dimensional arrays are

$$
\begin{gathered}
\bar{h}_{i},\left(\frac{\partial \bar{h}}{\partial \varphi}\right)_{i},\left(\frac{\partial \bar{h}}{\partial \bar{t}}\right)_{i}, \bar{p}_{i}^{*}=\bar{p}_{i, j=19}^{*},\left(\frac{\partial \bar{p}^{*}}{\partial \varphi}\right)_{i}=\left(\frac{\partial \bar{p}^{*}}{\partial \varphi}\right)_{i, j=19},\left(\frac{\partial \bar{p}^{*}}{\partial \bar{z}}\right)_{i}=\left(\frac{\partial \bar{p}^{*}}{\partial \bar{z}}\right)_{i, j=19}, \\
\left(K_{3}\right)_{i},\left(K_{4}\right)_{i},\left(K_{6}\right)_{k},\left(K_{7}\right)_{k},\left(K_{9}\right)_{i},\left(K_{10}\right)_{k},\left(Q_{1}\right)_{i}=\left(Q_{1}\right)_{i, j=19},\left(Q_{2}\right)_{i}=\left(Q_{2}\right)_{i, j=19} .
\end{gathered}
$$

The two-dimensional arrays are

$$
\begin{gathered}
\bar{\mu}_{i, k}=\bar{\mu}_{i, j=19, k},\left(\bar{V}_{x}\right)_{i, k}=\left(\bar{V}_{x}\right)_{i, j=19, k},\left(\bar{V}_{z}\right)_{i, k}=\left(\bar{V}_{z}\right)_{i, j=19, k}, \\
\left(\frac{\partial \bar{V}_{x}}{\partial \bar{y}}\right)_{i, k}=\left(\frac{\partial \bar{V}_{x}}{\partial \bar{y}}\right)_{i, j=19, k},\left(\frac{\partial \bar{V}_{z}}{\partial \bar{y}}\right)_{i, k}=\left(\frac{\partial \bar{V}_{z}}{\partial \bar{y}}\right)_{i, j=19, k}, \bar{\mu}_{i, k}^{*}=\bar{\mu}_{i, j=19, k}^{*}, \\
\left(\bar{q}_{x y}\right)_{i, k}=\left(\bar{q}_{x y}\right)_{i, j=19, k},\left(\bar{q}_{z y}\right)_{i, k}=\left(\bar{q}_{z y}\right)_{i, j=19, k},(\bar{D} s)_{i, k}=(\bar{D} s)_{i, j=19, k},(\bar{D})_{i, k}=(\bar{D})_{i, j=19, k} .
\end{gathered}
$$

To construct the difference analogs of (14), we apply the Pismen-Reckford method [9-12] with a two-step difference scheme. Let us discretize (14), using for all derivatives the simplest central differences

$$
\frac{\partial \bar{T}}{\partial \varphi}=\frac{\bar{T}_{i+1}-\bar{T}_{i-1}}{2 \Delta_{\varphi}}, \quad \frac{\partial^{2} \bar{T}}{\partial \varphi^{2}}=\frac{\bar{T}_{i+1}-2 \bar{T}_{i}+\bar{T}_{i-1}}{\Delta_{\varphi}^{2}} .
$$

Firstly, consider the discretization scheme of the heat equation (8) for the bushing. Divide the time step into two half-steps (Figure 2):

Step 1 (by $\varphi$ )

$$
\frac{\bar{T}_{i, k}^{n+1 / 2}-\bar{T}_{i, k}^{n}}{\Delta_{\bar{t}} / 2}=K_{5} \cdot L_{y y}(T)_{i, k}^{n}+K_{6 k} \cdot L_{y}(T)_{i, k}^{n}+K_{7 k} \cdot L_{\varphi \varphi}(T)_{i, k}^{n+1 / 2} .
$$

Step 2 (by $y$ )

$$
\begin{gathered}
\frac{\bar{T}_{i, k}^{n+1}-\bar{T}_{i, k}^{n+1 / 2}}{\Delta_{\bar{t}} / 2}=K_{5} \cdot L_{y y}(T)_{i, k}^{n+1}+K_{6 k} \cdot L_{y}(T)_{i, k}^{n+1}+K_{7 k} \cdot L_{\varphi \varphi}(T)_{i, k}^{n+1 / 2}, \\
\frac{\bar{T}_{i, k}^{n+1 / 2}-\bar{T}_{i, k}^{n}}{\Delta_{\bar{t}} / 2}=K_{5} \frac{\bar{T}_{i, k+1}^{n}-2 \bar{T}_{i, k}^{n}+\bar{T}_{i, k-1}^{n}}{\Delta_{y}^{2}}+ \\
+K_{6 k} \frac{\bar{T}_{i, k+1}^{n}-\bar{T}_{i, k-1}^{n}}{2 \Delta_{y}}+K_{7 k} \frac{\bar{T}_{i+1, k}^{n+1 / 2}-2 \bar{T}_{i, k}^{n+1 / 2}+\bar{T}_{i-1, k}^{n+1 / 2}}{\Delta_{\varphi}^{2}} \\
\frac{\bar{T}_{i, k}^{n+1}-\bar{T}_{i, k}^{n+1 / 2}}{\Delta_{\bar{t}} / 2}=K_{5} \frac{\bar{T}_{i, k+1}^{n+1}-2 \bar{T}_{i, k}^{n+1}+\bar{T}_{i, k-1}^{n+1}}{\Delta_{y}^{2}}+ \\
+K_{6 k} \frac{\bar{T}_{i, k+1}^{n+1}-\bar{T}_{i, k-1}^{n+1}}{2 \Delta_{y}}+K_{7 k} \frac{\bar{T}_{i+1, k}^{n+1 / 2}-2 \bar{T}_{i, k}^{n+1 / 2}+\bar{T}_{i-1, k}^{n+1 / 2}}{\Delta_{\varphi}^{2}}
\end{gathered}
$$

Express (15), (16) as

$$
a_{1} \bar{T}_{i-1, k}^{n+1 / 2}+b_{1} \bar{T}_{i, k}^{n+1 / 2}+c_{1} \bar{T}_{i+1, k}^{n+1 / 2}=d_{1} \bar{T}_{i, k-1}^{n}+e_{1} \bar{T}_{i, k}^{n}+f_{1} \bar{T}_{i, k+1}^{n},
$$




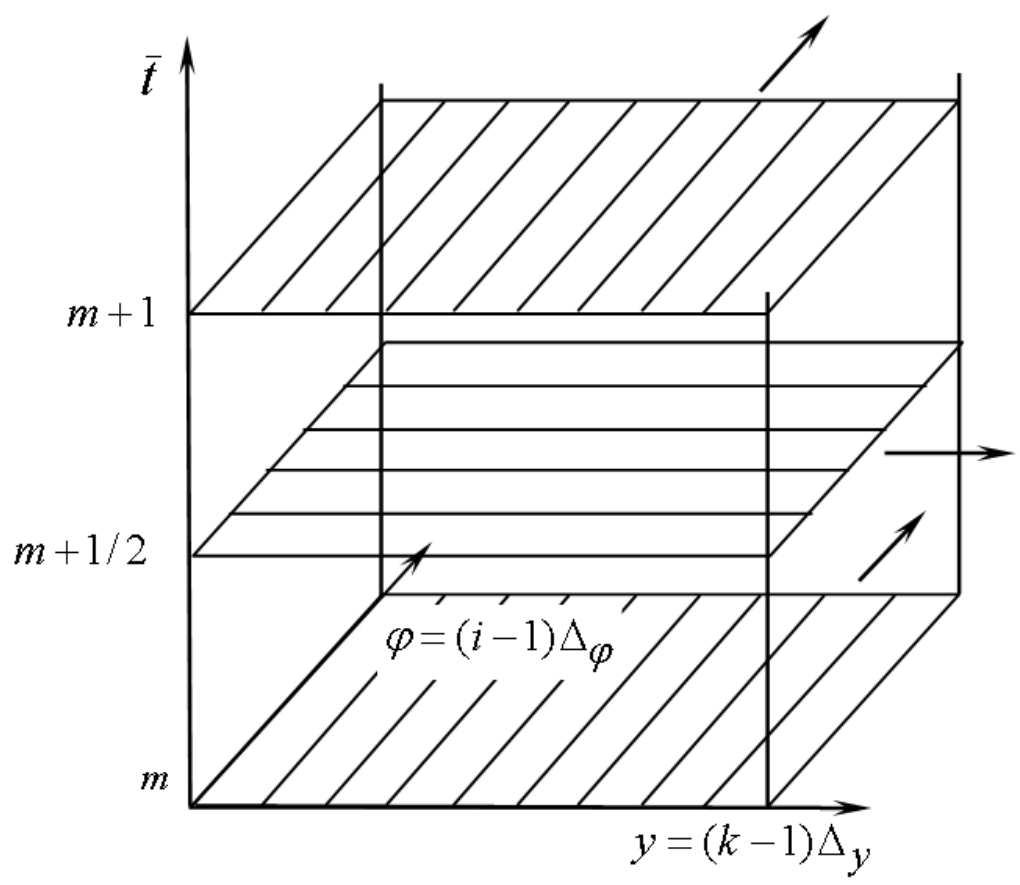

Fig. 2. Scheme of calculation by the implicit method of alternating directions. Arrows indicate the directions the scheme is implicit in

$$
a_{2} \bar{T}_{i, k-1}^{n+1}+b_{2} \bar{T}_{i, k}^{n+1}+c_{2} \bar{T}_{i, k+1}^{n+1}=d_{2} \bar{T}_{i, k-1}^{n+1 / 2}+e_{2} \bar{T}_{i, k}^{n+1 / 2}+f_{2} \bar{T}_{i, k+1}^{n+1 / 2}
$$

where

$$
\begin{gathered}
a_{1}=-\frac{K_{7 k}}{\Delta_{\varphi}^{2}}, b_{1}=2\left(\frac{1}{\Delta_{\bar{t}}}+\frac{K_{7 k}}{\Delta_{\varphi}^{2}}\right), c_{1}=-\frac{K_{7 k}}{\Delta_{\varphi}^{2}}, d_{1}=\left(\frac{K_{5}}{2 \Delta_{y}^{2}}-\frac{K_{6 k}}{2 \Delta_{y}}\right), e_{1}=2\left(-\frac{1}{\Delta_{\bar{t}}}-\frac{K_{5}}{\Delta_{y}^{2}}\right), \\
f_{1}=\frac{K_{5}}{\Delta_{y}^{2}}+\frac{K_{6 k}}{2 \Delta_{y}}, a_{2}=-\frac{K_{5}}{\Delta_{y}^{2}}+\frac{K_{6 k}}{2 \Delta_{y}}, b_{2}=2\left(\frac{1}{\Delta_{\bar{t}}}+\frac{K_{5}}{\Delta_{y}^{2}}\right), c_{2}=-\frac{K_{5}}{\Delta_{y}^{2}}-\frac{K_{6 k}}{2 \Delta_{y}}, d_{2}=\frac{K_{7 k}}{\Delta_{\varphi}^{2}}, \\
e_{2}=2\left(\frac{1}{\Delta_{\bar{t}}}+\frac{K_{7 k}}{\Delta_{\varphi}^{2}}\right), f_{2}=\frac{K_{7 k}}{\Delta_{\varphi}^{2}} .
\end{gathered}
$$

Similarly discretize the energy equation (5). Divide the time step into two halves: Step 1 (by $\varphi$ )

$$
\frac{\bar{T}_{i, k}^{n+1 / 2}-\bar{T}_{i, k}^{n}}{\Delta_{\bar{t}} / 2}=K_{3 i} \cdot L_{y y}(T)_{i, k}^{n}+K_{2 i, k}^{n} \cdot L_{y}(T)_{i, k}^{n}+K_{1 i, k}^{n} \cdot L_{\varphi}(T)_{i, k}^{n+1 / 2}+K_{4 i} .
$$

Step 2 (by $y$ )

$$
\frac{\bar{T}_{i, k}^{n+1}-\bar{T}_{i, k}^{n+1 / 2}}{\Delta_{\bar{t}} / 2}=K_{3 i} \cdot L_{y y}(T)_{i, k}^{n+1}+K_{2 i, k}^{n} \cdot L_{y}(T)_{i, k}^{n+1}+K_{1 i, k}^{n} \cdot L_{\varphi}(T)_{i, k}^{n+1 / 2}+K_{4 i}
$$

or

$$
\begin{aligned}
\frac{\bar{T}_{i, k}^{n+1 / 2}-\bar{T}_{i, k}^{n}}{\Delta_{\bar{t}} / 2}= & K_{3 i} \frac{\bar{T}_{i . k+1}^{n}-2 \bar{T}_{i, k}^{n}+\bar{T}_{i, k-1}^{n}}{\Delta_{y}^{2}}+K_{2 i, k}^{n} \frac{\bar{T}_{i, k+1}^{n}-\bar{T}_{i, k-1}^{n}}{2 \Delta_{y}}+ \\
& +K_{1 i, k}^{n} \frac{\bar{T}_{i+1, k}^{n+1 / 2}-\bar{T}_{i-1, k}^{n+1 / 2}}{2 \Delta_{\varphi}}+K_{4 i}
\end{aligned}
$$




$$
\begin{aligned}
\frac{\bar{T}_{i, k}^{n+1}-\bar{T}_{i, k}^{n+1 / 2}}{\Delta_{\bar{t}} / 2}= & K_{3} \frac{\bar{T}_{i, k+1}^{n+1}-2 \bar{T}_{i, k}^{n+1}+\bar{T}_{i, k-1}^{n+1}}{\Delta_{y}^{2}}+K_{2 i, k}^{n} \frac{\bar{T}_{i, k+1}^{n+1}-\bar{T}_{i, k-1}^{n+1}}{2 \Delta_{y}}+ \\
& +K_{1 i, k}^{n} \frac{\bar{T}_{i+1, k}^{n+1 / 2}-\bar{T}_{i-1, k}^{n+1 / 2}}{2 \Delta_{\varphi}}+K_{4 i} .
\end{aligned}
$$

Express $(19,20)$ as

$$
\begin{gathered}
a_{3} \bar{T}_{i-1, k}^{n+1 / 2}+b_{3} \bar{T}_{i, k}^{n+1 / 2}+c_{3} \bar{T}_{i+1, k}^{n+1 / 2}=d_{3} \bar{T}_{i, k-1}^{n}+e_{3} \bar{T}_{i, k}^{n}+f_{3} \bar{T}_{i, k+1}^{n}+g_{3}, \\
a_{4} \bar{T}_{i, k-1}^{n+1}+b_{4} \bar{T}_{i, k}^{n+1}+c_{4} \bar{T}_{i, k+1}^{n+1}=d_{4} \bar{T}_{i-1, k}^{n+1 / 2}+e_{4} \bar{T}_{i, k}^{n+1 / 2}+f_{4} \bar{T}_{i+1, k}^{n+1 / 2}+g_{4},
\end{gathered}
$$

where

$$
\begin{gathered}
a_{3}=\frac{K_{1 i, k}^{n}}{2 \Delta_{\varphi}}, b_{3}=\frac{2}{\Delta_{\bar{t}}}, c_{3}=-a_{3}, d_{3}=\frac{K_{3 i}}{\Delta_{y}^{2}}-\frac{K_{2 i, k}^{n}}{2 \Delta_{y}}, e_{3}=2\left(\frac{1}{\Delta_{\bar{t}}}-\frac{K_{3 i}}{\Delta_{y}^{2}}\right) \\
f_{3}=\frac{K_{3 i}}{\Delta_{y}^{2}}+\frac{K_{2 i, k}^{n}}{2 \Delta_{y}}, g_{3}=K_{4 i}, a_{4}=-\frac{K_{3 i}}{\Delta_{y}^{2}}+\frac{K_{2 i, k}^{n}}{2 \Delta_{y}}, b_{4}=2\left(\frac{1}{\Delta_{\bar{t}}}+\frac{K_{3 i}}{\Delta_{y}^{2}}\right), c_{4}=-\frac{K_{3 i}}{\Delta_{y}^{2}}-\frac{K_{2 i, k}^{n}}{2 \Delta_{y}}, \\
d_{4}=-\frac{K_{1 i, k}^{n}}{2 \Delta_{\varphi}}, e_{4}=\frac{2}{\Delta_{\bar{t}}}, f_{4}=\frac{K_{1 i, k}^{n}}{2 \Delta_{\varphi}}, g_{4}=K_{4 i} .
\end{gathered}
$$

The discretization of the heat equation (10) for the pin follows the same pattern.

Step 1 (by $\varphi$ )

$$
\frac{\bar{T}_{i, k}^{n+1 / 2}-\bar{T}_{i, k}^{n}}{\Delta_{\bar{t}} / 2}=K_{8} \cdot L_{y y}(T)_{i, k}^{n}+K_{9 k} \cdot L_{y}(T)_{i, k}^{n}+K_{10 k} \cdot L_{\varphi \varphi}(T)_{i, k}^{n+1 / 2} .
$$

Step 2 (by $y$ )

$$
\begin{gathered}
\frac{\bar{T}_{i, k}^{n+1}-\bar{T}_{i, k}^{n+1 / 2}}{\Delta_{\bar{t}} / 2}=K_{8} \cdot L_{y y}(T)_{i, k}^{n+1}+K_{9 k} \cdot L_{y}(T)_{i, k}^{n+1}+K_{10 k} \cdot L_{\varphi \varphi}(T)_{i, k}^{n+1 / 2}, \\
\frac{\bar{T}_{i, k}^{n+1 / 2}-\bar{T}_{i, k}^{n}}{\Delta_{\bar{t}} / 2}=K_{8} \frac{\bar{T}_{i, k+1}^{n}-2 \bar{T}_{i, k}^{n}+\bar{T}_{i, k-1}^{n}}{\Delta_{y}^{2}}+ \\
+K_{9 k} \frac{\bar{T}_{i, k+1}^{n}-\bar{T}_{i, k-1}^{n}}{2 \Delta_{y}}+K_{10 k} \frac{\bar{T}_{i+1, k}^{n+1 / 2}-2 \bar{T}_{i, k}^{n+1 / 2}+\bar{T}_{i-1, k}^{n+1 / 2}}{\Delta_{\varphi}^{2}} \\
\frac{\bar{T}_{i, k}^{n+1}-\bar{T}_{i, k}^{n+1 / 2}}{\Delta_{\bar{t}} / 2}=K_{8} \frac{\bar{T}_{i, k+1}^{n+1}-2 \bar{T}_{i, k}^{n+1}+\bar{T}_{i, k-1}^{n+1}}{\Delta_{y}^{2}}+ \\
+K_{9 k} \frac{\bar{T}_{i, k+1}^{n+1}-\bar{T}_{i, k-1}^{n+1}}{2 \Delta_{y}}+K_{10 k} \frac{\bar{T}_{i+1, k}^{n+1 / 2}-2 \bar{T}_{i, k}^{n+1 / 2}+\bar{T}_{i-1, k}^{n+1 / 2}}{\Delta_{\varphi}^{2}}
\end{gathered}
$$

Express the latter equations as

$$
\begin{gathered}
a_{5} \bar{T}_{i-1, k}^{n+1 / 2}+b_{5} \bar{T}_{i, k}^{n+1 / 2}+c_{5} \bar{T}_{i+1, k}^{n+1 / 2}=d_{5} \bar{T}_{i, k-1}^{n}+e_{5} \bar{T}_{i, k}^{n}+f_{5} \bar{T}_{i, k+1}^{n}, \\
a_{6} \bar{T}_{i, k-1}^{n+1}+b_{6} \bar{T}_{i, k}^{n+1}+c_{6} \bar{T}_{i, k+1}^{n+1}=d_{6} \bar{T}_{i, k-1}^{n+1 / 2}+e_{6} \bar{T}_{i, k}^{n+1 / 2}+f_{6} \bar{T}_{i, k+1}^{n+1 / 2},
\end{gathered}
$$


where

$$
\begin{gathered}
a_{5}=-\frac{K_{10 k}}{\Delta_{\varphi}^{2}}, b_{5}=2\left(\frac{1}{\Delta_{\bar{t}}}+\frac{K_{10 k}}{\Delta_{\varphi}^{2}}\right), c_{5}=-\frac{K_{10 k}}{\Delta_{\varphi}^{2}}, d_{5}=\left(\frac{K_{8}}{2 \Delta_{y}^{2}}-\frac{K_{9 k}}{2 \Delta_{y}}\right), \\
e_{5}=2\left(-\frac{1}{\Delta_{\bar{t}}}-\frac{K_{8}}{\Delta_{y}^{2}}\right), f_{5}=\frac{K_{8}}{\Delta_{y}^{2}}+\frac{K_{9 k}}{2 \Delta_{y}}, a_{6}=-\frac{K_{8}}{\Delta_{y}^{2}}+\frac{K_{9 k}}{2 \Delta_{y}}, b_{6}=2\left(\frac{1}{\Delta_{\bar{t}}}+\frac{K_{8}}{\Delta_{y}^{2}}\right), \\
c_{6}=-\frac{K_{8}}{\Delta_{y}^{2}}-\frac{K_{9 k}}{2 \Delta_{y}}, d_{6}=\frac{K_{10 k}}{\Delta_{\varphi}^{2}}, e_{6}=2\left(\frac{1}{\Delta_{\bar{t}}}+\frac{K_{10 k}}{\Delta_{\varphi}^{2}}\right), f_{6}=\frac{K_{10 k}}{\Delta_{\varphi}^{2}}
\end{gathered}
$$

Write down the groups of equations (17) and (18), (21) and (22), (23) and (24) at all internal nodes of the grid for all $\bar{t}>0$. This splitting reduces the problem to systems of algebraic equations with tridiagonal matrices. At step 1 we solve the system for each row (the series of points with fixed $k$ ) and at step 2 we solve the system for each column (the series of points with fixed $i$ ).

We begin by integrating the energy equation with the boundary conditions reflecting the frequency of temperature change in the circumferential direction and the equality of temperatures on the surfaces of the lubricating layer shared with the journal (the Dirichlet conditions). Their difference analogs are

$$
\bar{T}_{i-1, k}^{n}=\bar{T}_{i, k}^{n}, \bar{T}_{i-1, k}^{n+1 / 2}=\bar{T}_{i, k}^{n+1 / 2}, \bar{T}_{i-1, k}^{n+1}=\bar{T}_{i, k}^{n+1}, \bar{T}_{i, 1}^{n}=\bar{T}_{1 i, 1}^{\left(\tau-\tau_{c}\right)}, \bar{T}_{i, k}^{n}=\bar{T}_{2}^{\left(\tau-\tau_{c}\right)} .
$$

Applying the sweep method first in the circumferential direction, we write the recurrence

$$
\bar{T}_{i+1, k}^{n+1 / 2}=\xi_{i+1, j} \bar{T}_{i, k}^{n+1 / 2}+\eta_{i+1, k} .
$$

Using the formulas of the left sweep, find the coefficients $\xi_{i+1}$ and $\eta_{i+1}$ for all $i$ and $k$, then perform the sweep in the radial direction.

The experience of many researchers has shown that implicit schemes of the method of alternating directions allow for big time intervals and speed up calculations in general. Douglas [10] successfully implemented a three-step scheme to integrate this kind of equation and showed that the scheme of the second order of accuracy $O\left(\Delta_{t}^{2}, \Delta_{x}^{2}, \Delta_{y}^{2}, \Delta_{z}^{2}\right)$ is certainly stable.

\section{The Results}

The first results concern the dynamics of the connecting rod bearing of an internal combustion engine of type 13/15.

During the calculation, at each point in time we obtain the three-dimensional distribution of temperature in the lubricant. The distribution of temperature along the $\bar{y}$ coordinate is shown in Figure 3, where for each element of the system "journal - lubricating layer - bushing" we chose 20 mesh elements.

The solution rests on a finite difference approximation. We neglected journal tilting. Solving the equation for hydrodynamic pressure, we allow the viscosity of the lubricant to depend on the second invariant of the shear rate and the resulting temperature distribution. Calculations show that the maximal temperature of the lubricating layer is found in the region of the largest hydrodynamic pressures. 


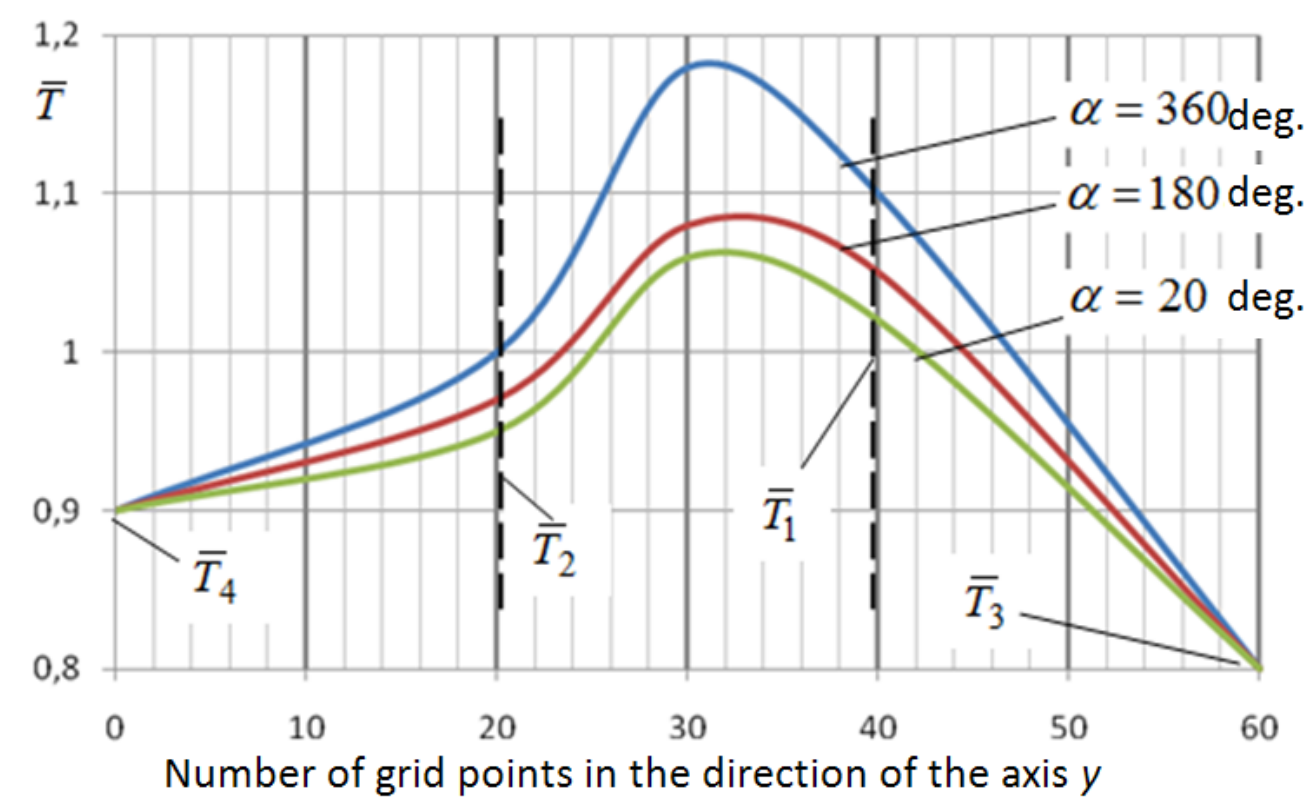

Fig. 3. The distribution of temperature along the $y$ coordinate

The results show that the heat exchange processes between the lubricating layer and the surrounding surfaces with convective heat transfer in the radial direction (see Fig. 3) reduce the mean temperature of the lubricant by 5 to 7 degrees. These results agree with the data of $[13,14]$.

\section{Conclusions}

(1) We presented a model and an algorithm for calculating the thermal hydrodynamic characteristics of heavily loaded bearings. They enable us to account for the processes of heat exchange between the lubricating layer with the properties of a non-Newtonian liquid and the elements of tribo-units.

(2) We proposed a solution algorithm based on a two-step difference approximation and an implicit scheme of the method of alternating directions, using which we can significantly increase the time intervals and decrease the running time of the calculation.

(3) The results show that in the design of heavily loaded tribo-units the models and algorithms we developed enable us to account for the rheological properties of the lubricant, the temperature distribution in the lubricating layer, and the thermal state of tribo-units as a whole.

Acknowledgements. This work was supported by a grant of the Ministry of Education and Science of the Russian Federation for applied research, code 2014-14-579-0109. The unique identifier for Applied Scientific Research (project) is RFMEFI57714X0102. Agreement no. 14.577.21.0102.

\section{References}

1. Prokopiev V.N., Karavaev V.G. [Task of Thermohydrodynamic Lubrication of Complex Loaded of Sliding Supports with non-Newtonian Fluids]. Vestnik YUrGU. Serya Mashinostroenie, 2003, no. 1 (17), pp. 55-66. (in Russian). 
2. Rozhdestvensky Y.V., Gavrilov K.V., Doikin A.A., Makarihin M.A. [Solution of Optimization Problems for Tribosystems of Piston and Rotary Machines Using the Mass Conservation Algorithm]. Trenie i Smazka v Mashinah i Mehanizmah, 2011, no. 8, pp. 38-43. (in Russian).

3. Zadorozhnaya E.A., Levanov I.G., Pyriev S.A. [Calculation of Complex Loaded Tribounits of High Performance Diesel]. Vestnik Mashinostroenia, 2013, no. 12, pp. 42-47. (in Russian).

4. Prokopiev V.N., Boyarshinova A.K., Zadorozhnaya E.A. Multigrid [Algorithms for Integrating of the Reynolds Equation in the Dynamics Complex Loaded Sliding Bearings]. Problemy Mashinostroenia i Nnadezhnosti Mashin, 2005, no. 5, pp. 16-21. (in Russian).

5. Levanov I.G. [Method of Calculation of Hydromechanical Characteristics of Piston and Rotary Machines Sliding Bearings with non-Newtonian Fluids]. Vestnik YUrGU. Serya Mashinostroenie, 2011, no. 31 (258), pp. 34-43. (in Russian).

6. Zadorozhnaya E.A. [Features of Modelling for Tribosystems of Pston and Rotary Machines Based on the Properties of the Lubricant]. Izvestiya Samarskogo Nauchnogo Centra RAN. Mehanika i Mashinostroenie, 2011, vol. 13, no. 4 (3), pp. 733-738. (in Russian)

7. Malenko P.I., Zelenko V.K., Levin D.M. Temperature Fields and Performance of the Pairs of Sliding Friction with a Lubricant. Edited by Y.N. Drozdov. Moscow, Mashinostroenie, 2011. 239 p.

8. Zhang C. TEHD Behavior of Non- Newtonian Dynamically Loadet Journal Bearings in Mixed Lubrication for Direct Problem. ASME Journal of Tribology, 2002, vol. 124, pp. $178-185$. DOI: $10.1115 / 1.1396342$

9. Rouch P. Computational Hydrodynamic. Moscow, Mir, 1980. 618 p.

10. Douglas J., Rachford H. H. On the Numerical Solution of Heat Conduction Problems in Two and Three Space Variables. Trans. Amer. Math. Soc., 1956, vol. 82, pp. 421439. DOI: $10.1090 /$ S0002-9947-1956-0084194-4

11. Ch Gao, Wang Y. A General Formulation of Peaceman and Richford ADI Method for the N-dimensional Heat Diffusion Equation. Int. Comm. Heat Mass Transfer, 1996, vol. 23, no. 6, pp. 845-854. DOI: 10.1016/0735-1933(96)00067-X

12. Samarsky A.A. The Theory of Difference Schemes. Moscow, Nauka, 1977, 653 p.

13. Zaharov S.M., Sirotenko V.I., Zharov I.A. [Simulation of Tribosystem "CrankshaftBearing - Supports of Cylinder Block" of Internal Combustion Engines]. Trenie $i$ Iznos, 1995, vol. 16, no. 1, pp. 47-54. (in Russian).

14. Chichinadze A.V., Berliner E.M., Braun E.D. Trenie, Iznos i Smazka: Tribologiya $i$ Tribotehnika. Edited by A.V. Chichinadze. Moscow, Mashinostroenie, 2003. 575 p.

Received September 25, 2014 
УДК 51-74; 532.5: 532.135; 621.822

DOI: $10.14529 / \operatorname{mmp} 140404$

\title{
МОДЕЛИРОВАНИЕ И ОЦЕНКА ТЕПЛОВОГО СОСТОЯНИЯ СЛОЖНОНАГРУЖЕННЫХ ТРИБОСОПРЯЖЕНИЙ
}

\author{
Ю.В. Рождественский, Е.А. Задорожсная
}

Тепловое состояние элементов сложнонагруженных трибосопряжений является одним из наиболее значимых параметров, влияющих на их работоспособность. Температура смазочного слоя подшипников скольжения во многом определяет его несущую способность. Использование уравнения теплового баланса для оценки температуры трибосопряжений не позволяет найти поля температур и зоны их максимальных значений. Этим определяется актуальность задачи. В статье рассмотрена математическая модель и методика расчета теплового состояния и термогидродинамических характеристик сложнонагруженных опор скольжения. При этом учитываются неньютоновские свойства жидкости, процессы теплообмена между смазочным слоем и окружающими его подвижными поверхностями трибосопряжения. Для решения уравнения энергии предложено использовать конечно-разностные аппроксимации. При построении разностных аналогов уравнений энергии для отдельных элементов конструкции и тонкого смазочного слоя был применен неявный метод переменных направлений ПисменаРекфорда. Приведены результаты расчета гидромеханических характеристик шатунного подшипника теплового двигателя. В процессе расчета были получены трехмерные распределения температуры в смазочном материале.

Результаты показали, что при учете конвективного переноса тепла в радиальном направлении, процессы теплообмена между смазочным слоем и окружающими его подвижными поверхностями дают возможность более точно определить среднеинтегральную температуру смазочного материала и теплонапряженность сопряжения в целом. Разработанная методика может быть использована при оценке характеристик и работоспособности сложнонагруженных трибосопряжения поршневых и роторных машин различного назначения.

Ключевые слова: опора жидкостного трения; обобщенное уравнение энергии; уравнения с частными производными; краевые задачи.

\section{Литература}

1. Прокопьев, В.Н. Термогидродинамическая задача смазки сложнонагруженных опор скольжения неньютоновскими жидкостями / В.Н. Прокопьев, В.Г. Караваев // Вестник ЮУрГУ. Серия: Машиностроение. - 2003. - № 1 (17). - С. 55-66.

2. Рождественский, Ю.В. Решение задач оптимизации трибосопряжений поршневых и роторных машин с использованием алгоритма сохранения массы / Ю.В. Рождественский, К.В. Гаврилов, А.А. Дойкин, М.А. Макарихин // Трение и смазка в машинах и механизмах. - 2011. - № 8. - С. 38-43.

3. Задорожная, Е.А. Расчет сложнонагруженных трибосопряжений высокофорсированного дизеля / Е.А. Задорожная, И.Г. Леванов, С.А. Пырьев // Вестник машиностроения. - 2013. - № 12. - С. 42-47.

4. Прокопьев, В.Н. Многосеточные алгоритмы интегрирования уравнения Рейнольдса в задачах динамики сложнонагруженных подшипников скольжения/ В.Н. Прокопьев, А.К. Бояршинова, Е.А. Задорожная // Проблемы машиностроения и надежности машин. - 2005. - № 5. - С. 16-21. 
5. Леванов, И.Г. Методика расчета гидромеханических характеристик сложнонагруженных подшипников скольжения поршневых и роторных машин, смазываемых неньютоновскими маслами / И.Г. Леванов // Вестник ЮУрГУ. Серия: Машиностроение. - 2011. - № 31 (258). - С. 34-43.

6. Задорожная, Е.А. Особенности моделирования трибосопряжений поршневых и роторных машин с учетом свойств смазочного материала // Е.А. Задорожная / Известия Самарского научного центра РАН. Механика и машиностроение. - 2011. - T. 13, №4 (3). - C. 733-738.

7. Маленко, П.И. Температурные поля и эксплуатационные свойства пар трения скольжения со смазочным материалом / П.И. Маленко, В.К. Зеленко, Д.М. Левин. Под ред. Ю.Н. Дроздова. - М.: Машиностроение. - 2011. - 239 с.

8. Zhang, C. TEHD Behavior of Non- Newtonian Dynamically Loadet Journal Bearings in Mixed Lubrication for Direct Problem / C. Zhang // ASME Journal of Tribology. - 2002. - V. 124. - P. 178-185.

9. Роуч, П. Вычислительная гидродинамика / П. Роуч. - М.: Мир, 1980. - 618 с.

10. Douglas, J. On the numerical solution of heat conduction problems in two and three space variables / J. Douglas, H. H. Rachford // Trans. Amer. Math. Soc. - 1956. V. 82. - P. 421-439.

11. Gao, Ch. A general formulation of Peaceman and Richford ADI method for the Ndimensional heat diffusion equation. / Ch. Gao, Y. Wang // Int. Comm. Heat Mass Transfer. - 1996. - V. 23. - №6. - P. 845-854.

12. Самарский, А.А. Теория разностных схем / А.А. Самарский. - М.: Наука, 1977. -653 c.

13. Захаров, С.М. Моделирование работы трибосистемы «коленчатый валподшипники - опоры блока цилиндров» двигателей внутреннего сгорания/ С.М. Захаров, В.И. Сиротенко, И.А. Жаров // Трение и износ. $-1995 .-$ Т. 16. №1. - C. 47-54.

14. Чичинадзе, А.В. Трение, износ и смазка: Трибология и триботехника / А.В. Чичинадзе, Э.М. Берлинер, Э.Д. Браун и др.; Под общ.ред. А.В. Чичинадзе// М.: Машиностроение, 2003. - 575 с.

Юрий Владимирович Рождественский, доктор технических наук, профессор, кафедра «Автомобильный транспорт и сервис автомобилей», Южно-Уральский государственный университет (г. Челябинск, Российская Федерация), ruv@susu.ac.ru.

Елена Анатольевна Задорожная, доктор технических наук, доцент, кафедра «Автомобильный транспорт и сервис автомобилей», Южно-Уральский государственный университет (г. Челябинск, Российская Федерация), elena-nea@rambler.ru.

Поступила в редакцию 25 сентября 20142. 